Illinois Journal of Mathematics

Volume 46, Number 3, Fall 2002, Pages 857-883

S $0019-2082$

\title{
NONLINEAR POTENTIAL THEORY ON METRIC SPACES
}

\author{
JUHA KINNUNEN AND OLLI MARTIO
}

\begin{abstract}
We study nonlinear potential theory on a metric measure space equipped with a doubling measure and supporting a Poincaré inequality. Minimizers, superminimizers and the obstacle problem for the $p$-Dirichlet integral play an important role in the theory. We prove lower semicontinuity of superminimizers and continuity of the solution to the obstacle problem with a continuous obstacle. We also show that the limit of an increasing sequence of superminimizers is a superminimizer provided it is bounded above. Moreover, we consider superharmonic functions and study their relations to superminimizers. Our proofs are based on the direct methods of the calculus of variations and on De Giorgi type estimates. In particular, we do not use the Euler-Lagrange equation and our arguments are based on the variational integral only.
\end{abstract}

\section{Introduction}

Let $(X, d)$ be a metric space equipped with a Borel measure $\mu$. In a metric measure space the concept of an upper gradient serves as a substitute for the Sobolev gradient. Suppose that $1 \leq p<\infty$ and let $u$ be a real-valued function on $X$. A non-negative Borel measurable function $g$ on $X$ is said to be a $p$-weak upper gradient of $u$ if for $p$-almost every rectifiable path $\gamma$ joining two points $x$ and $y$ in $X$ we have

$$
|u(x)-u(y)| \leq \int_{\gamma} g d s .
$$

This means that (1.1) holds for all paths in $X$ outside a family of paths which is of $p$-modulus zero. The Sobolev space on a metric measure space, called the Newtonian space $N^{1, p}(X)$, can be defined as a collection of equivalence classes of $p$-integrable functions with $p$-integrable upper gradients. Essentially, this is an extension to metric spaces of the absolute continuity property on almost all lines of Sobolev functions. For this approach on a metric measure space see [Sh2]; a slightly different, but equivalent approach is used in [C].

Received January 3, 2002; received in final form May 8, 2002.

2000 Mathematics Subject Classification. 46E35, 31C15. 
Newtonian spaces enable us to study variational integrals on a metric measure space, and nonlinear potential theoretic models can be built on minimizers of variational integrals

$$
\int g_{u}^{p} d \mu
$$

where $g_{u}$ denotes the minimal $p$-weak upper gradient of $u$. Indeed, in [KS] it was shown that the minimizers, and even the quasiminimizers, of (1.2) satisfy the Harnack inequality and the maximum principle, and are locally Hölder continuous under relatively mild conditions. For recent developments in the analysis on metric spaces we refer to [B], [BBS], [BMS], [C], [Ha], [HeK], [Sh1], [Sh2]. A very nice general overview of the theory can be found in $[\mathrm{HaK}]$ and [He].

The objective of this paper is to study nonlinear potential theory on a metric measure space. Due to the notion of the upper gradient, it is not clear how to employ partial differential equations in this setting. Our approach is based only on the variational integrals (1.2), and therefore new arguments are needed. In contrast to the potential theory of minimizers of the $p$-Dirichlet integral in the Euclidean case, which is nonlinear only when $p \neq 2$, our theory is nonlinear for all $p>1$. The reason for this is that the operation of taking the upper gradient is not linear. Classical harmonic functions are replaced by continuous minimizers of (1.2). Superminimizers and the obstacle problem play an important role in the theory. We prove the lower semicontinuity of superminimizers and the continuity of the solution to the obstacle problem with a continuous obstacle. We also show that the limit of an increasing sequence of superminimizers is a superminimizer provided it is bounded above. Our proofs are based on direct methods of the calculus of variations and on De Giorgi type estimates; see [Gia], [Giu]. Traditionally these estimates have been used to prove local Hölder continuity and the Harnack inequality for minimizers of variational integrals. We use them to handle semicontinuity problems associated with super- and subminimizers. We close the paper by considering superharmonic functions and studying their relations to superminimizers.

In a forthcoming paper the authors study further properties of nonlinear potential theory on metric measure spaces and show that much of the theory can be extended to quasiminimizers. Methods like the Poisson modification and the Perron method seem to be available also in the metric setting, and they open possibilities to study more refined aspects of nonlinear potential theory on metric measure spaces.

\section{Newtonian spaces}

Let $X$ be a metric space and let $\mu$ be a Borel measure on $X$. Throughout the paper we assume that the measure of every nonempty open set is positive 
and that the measure of every bounded set is finite. Later we will impose further requirements on the space and on the measure (see 2.14).

2.1. Upper gradients. Let $u$ be a real-valued function on $X$. A nonnegative Borel measurable function $g$ on $X$ is said to be an upper gradient of $u$ if for all rectifiable paths $\gamma$ joining points $x$ and $y$ in $X$ we have

$$
|u(x)-u(y)| \leq \int_{\gamma} g d s .
$$

See $[\mathrm{C}],[\mathrm{He}],[\mathrm{HeK}]$ and $[\mathrm{Sh} 1]$ for a discussion of upper gradients.

Let $1 \leq p<\infty$. The $p$-modulus of a family of paths $\Gamma$ in $X$ is the number

$$
\inf _{\rho} \int_{X} \rho^{p} d \mu,
$$

where the infimum is taken over all non-negative Borel measurable functions $\rho$ such that for all rectifiable paths $\gamma$ which belong to $\Gamma$ we have

$$
\int_{\gamma} \rho d s \geq 1 .
$$

It is known that the $p$-modulus is an outer measure on the collection of all paths in $X$.

A property is said to hold for $p$-almost all paths, if the set of paths for which the property fails is of zero $p$-modulus. If $(2.2)$ holds for $p$-almost all paths $\gamma$, then $g$ is said to be a $p$-weak upper gradient of $u$.

2.3. Newtonian spaces. Let $1 \leq p<\infty$. We define the space $\widetilde{N}^{1, p}(X)$ to be the collection of all $p$-integrable functions $u$ on $X$ that have a $p$-integrable $p$-weak upper gradient $g$ on $X$. This space is equipped with a seminorm

$$
\|u\|_{\widetilde{N}^{1, p}(X)}=\|u\|_{L^{p}(X)}+\inf \|g\|_{L^{p}(X)},
$$

where the infimum is taken over all $p$-weak upper gradients of $u$.

We define an equivalence relation in $\widetilde{N}^{1, p}(X)$ by saying that $u \sim v$ if

$$
\|u-v\|_{\widetilde{N}^{1, p}(X)}=0 .
$$

The Newtonian space $N^{1, p}(X)$ is defined to be the space $\tilde{N}^{1, p}(X) / \sim$ with the norm

$$
\|u\|_{N^{1, p}(X)}=\|u\|_{\widetilde{N}^{1, p}(X)} \cdot
$$

For basic properties of the Newtonian spaces we refer to [Sh1]. Cheeger $[\mathrm{C}]$ gives an alternative definition which leads to the same space when $1<p<\infty$; see [Sh1].

For future reference we recall some known facts: 
(i) The functions in $\widetilde{N}^{1, p}(X)$ are defined outside a path family of $p$ modulus zero. This implies that functions in $\widetilde{N}^{1, p}(X)$ cannot be arbitrarily changed on sets of measure zero. However, if $u, v \in \widetilde{N}^{1, p}(X)$ such that $u=v \mu$-almost everywhere, then $u$ and $v$ belong to the same equivalence class in $N^{1, p}(X)$.

(ii) The normed space $\left(N^{1, p}(X),\|\cdot\|_{N^{1, p}(X)}\right)$ is complete, i.e., it is a Banach space.

(iii) If $1<p<\infty$, every function $u$ that has a $p$-integrable $p$-weak upper gradient has a minimal $p$-integrable $p$-weak upper gradient in $X$, denoted by $g_{u}$, in the sense that if $g$ is another $p$-weak upper gradient of $u$, then $g_{u} \leq g \mu$-almost everywhere in $X$.

(iv) For every $c \in \mathbf{R}$ the minimal $p$-weak upper gradient satisfies $g_{u}=0 \mu$ almost everywhere on the set $\{x \in X: u(x)=c\}$. This implies that if $u, v \in N^{1, p}(X)$, then $\min (u, v) \in N^{1, p}(X)$ and $\max (u, v) \in N^{1, p}(X)$. Moreover, if $g_{u}$ and $g_{v}$ are the minimal $p$-weak upper gradients of $u$ and $v$, respectively, then $g_{u} \chi_{E}+g_{v} \chi_{X \backslash E}$, with $E=\{x \in X: u(x) \geq$ $v(x)\}$, is a $p$-weak upper gradient of $\min (u, v)$.

(v) If $u \in N^{1, p}(X)$ and $v$ is a bounded Lipschitz continuous function, then $u v \in N^{1, p}(X)$ and $g_{u v} \leq|u| g_{v}+|v| g_{u} \mu$-almost everywhere.

(vi) Suppose that $u_{i}, i=1,2, \ldots$, is a sequence of functions in $N^{1, p}(X)$, $1<p<\infty$, such that

$$
\sup _{i}\left\|u_{i}\right\|_{N^{1, p}(X)}<\infty .
$$

Then there is a subsequence $u_{i_{j}}$ and a function $u \in N^{1, p}(X)$ such that $u_{i_{j}} \rightarrow u$ weakly in $L^{p}(X)$ and

$$
\left\|g_{u}\right\|_{L^{p}(X)} \leq \liminf _{j \rightarrow \infty}\left\|g_{u_{i_{j}}}\right\|_{L^{p}(X)}
$$

(vii) The functions in $N^{1, p}(X)$ are absolutely continuous on p-almost every path, i.e., $u \circ \gamma$ is absolutely continuous on $[0, \operatorname{length}(\gamma)]$ for $p$-almost every rectifiable arc-length parametrized path $\gamma$ in $X$.

We emphasize that these properties hold without any additional assumptions on the measure and on the space. The property (vi) would follow from the reflexivity of the Newtonian spaces $N^{1, p}(X), 1<p<\infty$. However, these spaces are known to be reflexive only under further assumptions on the measure and space; see $[\mathrm{C}]$. We stress that reflexivity is not needed in the proof of (vi).

Next we prove an estimate for the $p$-weak upper gradient of a certain function which will be used as a test function later. This estimate does not seem to follow from (v), and hence a more careful analysis is needed.

2.4. LemmA. Suppose that $u, v \in N^{1, p}(X)$ and that $\eta$ is a Lipschitz continuous function in $X$ with $0 \leq \eta \leq 1$ and $v \geq u \mu$-almost everywhere in $X$. Let $g_{u}, g_{v}$ and $g_{\eta}$ be the $p$-weak upper gradients of $u$, $v$ and $\eta$, respectively. 
Define $w=u+\eta(v-u)$. Then

$$
g_{w} \leq(1-\eta) g_{u}+\eta g_{v}+(v-u) g_{\eta}
$$

$\mu$-almost everywhere in $X$.

Proof. Let $\gamma$ be the arc-length parametrization of a rectifiable path on which the functions $u, v$ and $\eta$ are absolutely continuous. Define the function $h:[0, \operatorname{length}(\gamma)] \rightarrow[0, \infty)$ by

$$
h(s)=(u \circ \gamma)(s)+(\eta \circ \gamma)(s)((v \circ \gamma)(s)-(u \circ \gamma)(s)) .
$$

Then $h$ is absolutely continuous and for almost every $s \in[0$, length $(\gamma)]$, with respect to the one-dimensional Lebesgue measure, we have

$$
\begin{gathered}
h^{\prime}(s)=(u \circ \gamma)^{\prime}(s)(1-(\eta \circ \gamma)(s))+(\eta \circ \gamma)^{\prime}(s)((v \circ \gamma)(s)-(u \circ \gamma)(s)) \\
+(\eta \circ \gamma)(s)(v \circ \gamma)^{\prime}(s) .
\end{gathered}
$$

Since $\left|(u \circ \gamma)^{\prime}(s)\right| \leq g_{u}(\gamma(s)),\left|(v \circ \gamma)^{\prime}(s)\right| \leq g_{v}(\gamma(s))$ and $\left|(\eta \circ \gamma)^{\prime}(s)\right| \leq g_{\eta}(\gamma(s))$ for almost every $s \in[0$, length $(\gamma)]$, we obtain

$$
\begin{gathered}
\left|h^{\prime}(s)\right| \leq g_{u}(\gamma(s))(1-\eta(\gamma(s)))+g_{\eta}(\gamma(s))(v(\gamma(s))-u(\gamma(s))) \\
+\eta(\gamma(s)) g_{v}(\gamma(s))
\end{gathered}
$$

for almost every $s \in[0$, length $(\gamma)]$. This proves $(2.5)$.

2.6. Capacity. The p-capacity of a set $E \subset X$ is defined by

$$
\mathrm{C}_{p}(E)=\inf _{u}\|u\|_{N^{1, p}(X)}^{p},
$$

where the infimum is taken over all functions $u \in N^{1, p}(X)$, whose restriction to $E$ is bounded below by 1 . The discussion in $[\mathrm{KM}]$ can easily be adapted to show that the capacity is an outer measure; see also [Sh1]. A property is said to hold $p$-quasieverywhere, if it holds everywhere except on a set of $p$-capacity zero. A function is p-quasicontinuous, if there is an open set of arbitrarily small $p$-capacity such that the function is continuous when restricted to the complement of the set.

The $p$-capacity is the natural measure for exceptional sets of Sobolev functions. Every function in $\widetilde{N}^{1, p}(X)$ is defined $p$-quasieverywhere. Moreover, if $u, v \in N^{1, p}(X)$ and $u=v \mu$-almost everywhere, then $u=v p$-quasieverywhere. In particular, this implies that $u$ and $v$ belong to the same equivalence class in $N^{1, p}(X)$.

Suppose that $E \subset X$. Then $\Gamma_{E}$ denotes the family of all rectifiable paths that intersect the set $E$. It is possible to show that $\mathrm{C}_{p}(E)=0$ implies that the $p$-modulus of $\Gamma_{E}$ is zero; see [Sh1]. From this it follows that if $u \in N^{1, p}(X)$ and $v$ is a measurable function on $X$ such that $v=u p$-quasieverywhere, then $v$ belongs to the same equivalence class in $N^{1, p}(X)$. 
2.7. Newtonian spaces with zero boundary values. In order to be able to compare the boundary values of Sobolev functions we need a notion of Sobolev spaces with zero boundary values in a metric measure space. Let $E$ be an arbitrary subset of $X$. Following the method of [KKM], we define $\widetilde{N}_{0}^{1, p}(E)$ to be the set of functions $u: E \rightarrow[-\infty, \infty]$ for which there exists a function $\widetilde{u} \in \widetilde{N}^{1, p}(X)$ such that $\widetilde{u}=u \mu$-almost everywhere in $E$ and

$$
\mathrm{C}_{p}(\{x \in X \backslash E: \widetilde{u}(x) \neq 0\})=0 .
$$

Next we define an equivalence relation on $\widetilde{N}_{0}^{1, p}(E)$ by requiring that $u \sim v$ if $u=v \mu$-almost everywhere on $E$. Finally we let $N_{0}^{1, p}(E)=\widetilde{N}_{0}^{1, p}(E) / \sim$, equipped with the norm

$$
\|u\|_{N_{0}^{1, p}(E)}=\|\widetilde{u}\|_{\widetilde{N}^{1, p}(X)},
$$

be the Newtonian space with zero boundary values. By [Sh1], the norm is unambiguously defined and the space so obtained is a Banach space.

2.8. Local Newtonian spaces. We are mainly interested in the local properties of minimizers of variational integrals. Hence we introduce the notion of a local Newtonian space as follows. Let $\Omega$ be an open subset of $X$. We say that a subset $A$ of $\Omega$ is compactly contained in $\Omega$, and write $A \subset \subset \Omega$, if the closure of $A$ is a compact subset of $\Omega$.

We say that $u$ belongs to the local Newtonian space $N_{\text {loc }}^{1, p}(\Omega)$ if $u \in N^{1, p}(A)$ for every measurable set $A \subset \subset \Omega$. If $u \in N_{\text {loc }}^{1, p}(\Omega)$ with $1<p<\infty$, then $u$ has a minimal $p$-weak upper gradient $g_{u}$ in $\Omega$ in the following sense: If $\Omega^{\prime} \subset \subset \Omega$ is an open set and $g$ is the minimal upper gradient of $u$ in $\Omega^{\prime}$, then $g_{u}=g$ $\mu$-almost everywhere in $\Omega^{\prime}$.

2.9. Doubling property. The measure $\mu$ is doubling if there is a constant $c_{d} \geq 1$ so that

$$
\mu(B(z, 2 r)) \leq c_{d} \mu(B(z, r))
$$

for every open ball $B(z, r)$ in $X$. The constant $c_{d}$ is called the doubling constant of $\mu$. Note that by the doubling property, if $B(y, R)$ is a ball in $X$, $z \in B(y, R)$ and $0<r \leq R<\infty$, then

$$
\frac{\mu(B(z, r))}{\mu(B(y, R))} \geq c\left(\frac{r}{R}\right)^{Q}
$$

for $c=1 / 2$ and $Q=\log c_{d} / \log 2$.

A metric space $X$ is said to be doubling if there is a constant $c<\infty$ such that every ball $B(x, r), x \in X, r>0$, can be covered by at most $c<\infty$ balls of radius $r / 2$. If $X$ is equipped with a doubling measure, then $X$ is doubling. 
2.10. Poincaré inequalities. Let $1 \leq q<\infty$. The space $X$ is said to support a weak $(1, q)$-Poincaré inequality if there are constants $c>0$ and $\tau \geq 1$ such that

$$
f_{B(z, r)}\left|u-u_{B(z, r)}\right| d \mu \leq c r\left(f_{B(z, \tau r)} g^{q} d \mu\right)^{1 / q}
$$

for all balls $B(z, r)$ in $X$, all integrable functions $u$ in $B(z, \tau r)$ and all $q$-weak upper gradients $g$ of $u$. The word weak refers to the possibility that $\tau>1$. If $\tau=1$, the space is said to support a $(1, q)$-Poincaré inequality. In a doubling measure space a weak $(1, q)$-Poincaré inequality implies a weak $(t, q)$-Poincaré inequality for some $t>q$, possibly with a different value of $\tau$; see [HaK]. More precisely, there are $t>q, c>0$ and $\tau^{\prime} \geq 1$ such that

$$
\left(f_{B(z, r)}\left|u-u_{B(z, r)}\right|^{t} d \mu\right)^{1 / t} \leq c r\left(f_{B\left(z, \tau^{\prime} r\right)} g^{q} d \mu\right)^{1 / q}
$$

for all balls $B(z, r)$ in $X$, all integrable functions $u$ in $B(z, r)$ and all $p$-weak upper gradients $g$ of $u$.

In particular, if $X$ supports a weak $(1, q)$-Poincaré inequality for some $q$ with $1<q<p$, then by Hölder's inequality it supports a weak $(1, p)$-Poincaré inequality, and hence $[\mathrm{HaK}]$ implies that

$$
\left(f_{B(z, r)}\left|u-u_{B(z, r)}\right|^{p} d \mu\right)^{1 / p} \leq c r\left(f_{B\left(z, \tau^{\prime} r\right)} g^{p} d \mu\right)^{1 / p}
$$

for all balls $B(z, r)$ in $X$, all integrable functions $u$ in $B(z, r)$ and all $p$-weak upper gradients $g$ of $u$.

2.14. General setup. We make the following rather standard assumptions:

We assume, without further notice, that the complete metric measure space $X$ is equipped with a doubling Borel measure for which the measure of every nonempty open set is positive and the measure of every bounded set is finite. Furthermore we assume that the space supports a weak $(1, q)$-Poincaré inequality for some $q$ with $1<q<p$.

We recall a few useful properties of Newtonian spaces which hold under these assumptions.

(i) Closed and bounded sets are compact and $\mu$ is a regular Borel measure.

(ii) The different definitions of Sobolev spaces yield the same space.

(iii) The standard Sobolev embeddings hold in the Newtonian space. 
(iv) Lipschitz continuous functions are dense in $N^{1, p}(X)$ and Lipschitz continuous functions which vanish in the complement of $E$ are dense in $N_{0}^{1, p}(E)$.

(v) Every function in $N^{1, p}(X)$ is $p$-quasicontinuous.

(vi) If $u \in N^{1, p}(X)$, then

$$
\lim _{r \rightarrow 0} f_{B(x, r)} u d \mu=u^{*}(x)
$$

exists $p$-quasieverywhere and defines a $p$-quasicontinuous function.

See [Sh1] for the properties (i)-(v); for (vi) we refer to [KL]. The assumption on the Poincaré inequality is also needed in the regularity theory for quasiminimizers of variational integrals on metric spaces; see [KS].

\section{The obstacle problem}

Let $1<p<\infty$. Suppose that $\Omega \subset X$ is an open set, let $\vartheta \in N^{1, p}(\Omega)$ and let $\psi: \Omega \rightarrow[-\infty, \infty]$ be an arbitrary function. We define

$$
\mathcal{K}_{\psi, \vartheta}(\Omega)=\left\{v \in N^{1, p}(\Omega): v-\vartheta \in N_{0}^{1, p}(\Omega), v \geq \psi \mu \text {-a.e. in } \Omega\right\} .
$$

A function $u \in \mathcal{K}_{\psi, \vartheta}(\Omega)$ is a solution of the $\mathcal{K}_{\psi, \vartheta}(\Omega)$-obstacle problem with the obstacle $\psi$ and the boundary values $\vartheta$ if

$$
\int_{\Omega} g_{u}^{p} d \mu \leq \int_{\Omega} g_{v}^{p} d \mu
$$

for every $v \in \mathcal{K}_{\psi, \vartheta}(\Omega)$. Here $g_{u}$ and $g_{v}$ are the minimal $p$-weak upper gradients of $u$ and $v$ in $\Omega$, respectively. In other words, the solution of the obstacle problem minimizes the $p$-Dirichlet energy among all functions which lie above the obstacle function $\psi$ and have the boundary values $\vartheta$. If $\psi=-\infty$, then the obstacle does not represent a restriction and a solution of the $\mathcal{K}_{-\infty, \vartheta}(\Omega)$ obstacle problem is said to be a minimizer with boundary values $\vartheta$ in $\Omega$. In other words, it is a solution of the Dirichlet problem with the boundary values $\vartheta$ in $\Omega$.

Using the direct methods of the calculus of variations it is possible to prove that under very mild conditions the obstacle problem has a unique solution.

3.2. THEOREM. Suppose that $\Omega \subset X$ is a bounded open set such that $\mu(X \backslash \Omega)>0$. If $\mathcal{K}_{\psi, \vartheta}(\Omega) \neq \emptyset$, there is a unique solution of the $\mathcal{K}_{\psi, \vartheta}(\Omega)$ obstacle problem.

Proof. Set

$$
I=\inf \int_{\Omega} g_{v}^{p} d \mu,
$$

where the infimum is taken over all $v \in \mathcal{K}_{\psi, \vartheta}(\Omega)$. Since $\mathcal{K}_{\psi, \vartheta}(\Omega) \neq \emptyset$, we observe that $0 \leq I<\infty$. Let $u_{i} \in \mathcal{K}_{\psi, \vartheta}(\Omega), i=1,2, \ldots$, be a minimizing 
sequence such that

$$
I=\lim _{i \rightarrow \infty} \int_{\Omega} g_{u_{i}}^{p} d \mu .
$$

In particular, this implies that the sequence $g_{u_{i}}, i=1,2, \ldots$, is bounded in $L^{p}(\Omega)$. Since $\mu(X \backslash \Omega)>0$ and $\Omega$ is bounded, a version of the Poincaré inequality (Lemma 2.1 in $[\mathrm{KS}]$ ) implies that

$$
\int_{\Omega}\left|u_{i}-\vartheta\right|^{p} d \mu \leq c \int_{\Omega}\left|g_{u_{i}-\vartheta}\right|^{p} d \mu \leq c \int_{\Omega} g_{u_{i}}^{p} d \mu+c \int_{\Omega} g_{\vartheta}^{p} d \mu,
$$

from which it follows that

$$
\sup _{i}\left\|u_{i}-\vartheta\right\|_{N_{0}^{1, p}(\Omega)}<\infty
$$

Since the sequence $u_{i}-\vartheta, i=1,2, \ldots$, is uniformly bounded in $N_{0}^{1, p}(\Omega)$, there is a subsequence $u_{i_{j}}$ and a function $u \in N^{1, p}(\Omega)$ such that $u-\vartheta \in N_{0}^{1, p}(\Omega)$, $u_{i_{j}} \rightarrow u$ weakly in $L^{p}(\Omega)$ and

$$
\int_{\Omega} g_{u}^{p} d \mu \leq \liminf _{j \rightarrow \infty} \int_{\Omega} g_{u_{i_{j}}}^{p} d \mu \leq I
$$

see 2.3 (vi). Hence $u$ is the desired minimizer.

We next show that $u \in \mathcal{K}_{\psi, \vartheta}(\Omega)$. We already know that $u-\vartheta \in N_{0}^{1, p}(\Omega)$, so it is enough to prove that $u \geq \psi \mu$-almost everywhere in $\Omega$. The Mazur lemma implies that a sequence $v_{j}, j=1,2, \ldots$, of convex combinations of $u_{i_{j}}$ converges strongly to $u$ in $L^{p}(\Omega)$. Passing to a subsequence we may assume that the sequence $v_{j}$ converges to $u \mu$-almost everywhere in $\Omega$. Since $u_{i_{j}} \geq \psi$ $\mu$-almost everywhere in $\Omega$, we conclude that $v_{j} \geq \psi \mu$-almost everywhere in $\Omega$ for every $j=1,2, \ldots$, and consequently that $u \geq \psi \mu$-almost everywhere in $\Omega$.

To prove the uniqueness, suppose that $u_{1}, u_{2} \in \mathcal{K}_{\psi, \vartheta}(\Omega)$ are solutions. As in Theorem 7.14 of $[\mathrm{C}]$ we conclude that $g_{u_{1}-u_{2}}=0 \mu$-almost everywhere in $\Omega$, and the uniqueness follows from the Poincaré inequality.

We are mostly interested in local properties of solutions of the obstacle problem and especially in defining solutions locally without any requirements on the boundary values. To this end, let $\Omega \subset X$ be open. A function $u \in$ $N_{\text {loc }}^{1, p}(\Omega)$ is a minimizer in $\Omega$, if it is a solution of the $\mathcal{K}_{-\infty, u}\left(\Omega^{\prime}\right)$-obstacle problem for every open set $\Omega^{\prime} \subset \subset \Omega$. Similarly, a function $u \in N_{\text {loc }}^{1, p}(\Omega)$ is a superminimizer in $\Omega$, if it is a solution of the $\mathcal{K}_{u, u}\left(\Omega^{\prime}\right)$-obstacle problem for every open set $\Omega^{\prime} \subset \subset \Omega$. In the Euclidean case minimizers correspond to solutions and superminimizers correspond to supersolutions of the $p$-Laplace equation. Since we do not have the Euler-Lagrange equation available here, we have to base our definitions only on the variational integral. 
A solution of the $\mathcal{K}_{\psi, \vartheta}(\Omega)$-obstacle problem is a superminimizer, but the converse is not true in general. However, if both $u$ and $-u$ are superminimizers in $\Omega$, then $u$ is a minimizer in $\Omega$.

We observe that if $u$ is a superminimizer, then $\alpha u$ and $u+\beta$ are superminimizers whenever $\alpha \geq 0$ and $\beta \in \mathbf{R}$. However, the sum of superminimizers is in general not a superminimizer.

We now prove a useful result for superminimizers, which corresponds to the classical result that the minimum of two supersolutions is a supersolution.

3.3. LEMMA. If $u_{1}$ and $u_{2}$ are superminimizers in $\Omega$, then $\min \left(u_{1}, u_{2}\right)$ is a superminimizer in $\Omega$.

Proof. Let $u=\min \left(u_{1}, u_{2}\right)$. Since $N_{\text {loc }}^{1, p}(\Omega)$ is a lattice, we have $u \in$ $N_{\text {loc }}^{1, p}(\Omega)$. Let $\Omega^{\prime} \subset \subset \Omega$ be an open set and let $v \in N^{1, p}\left(\Omega^{\prime}\right)$, such that $u-v \in N_{0}^{1, p}\left(\Omega^{\prime}\right)$ and $v \geq u \mu$-almost everywhere in $\Omega^{\prime}$. Set $A=\left\{x \in \Omega^{\prime}\right.$ : $\left.u_{1}(x)>u_{2}(x)\right\}, B=\left\{x \in \Omega^{\prime}: v(x) \geq u_{1}(x)\right\}$ and $w=\max \left(\min \left(u_{1}, v\right), u_{2}\right)$. Then $w-u_{2} \in N_{0}^{1, p}\left(\Omega^{\prime}\right), w \geq u_{2} \mu$-almost everywhere in $\Omega^{\prime}$ and $w=u_{2}$ $\mu$-almost everywhere in $\Omega^{\prime} \backslash A$. By the superminimizing property of $u_{2}$ in $\Omega^{\prime}$ we obtain

$$
\begin{aligned}
\int_{A} g_{u_{2}}^{p} d \mu+\int_{\Omega^{\prime} \backslash A} g_{u_{2}}^{p} d \mu & =\int_{\Omega^{\prime}} g_{u_{2}}^{p} d \mu \leq \int_{\Omega^{\prime}} g_{w}^{p} d \mu \\
& =\int_{A} g_{w}^{p} d \mu+\int_{\Omega^{\prime} \backslash A} g_{w}^{p} d \mu \\
& =\int_{A} g_{w}^{p} d \mu+\int_{\Omega^{\prime} \backslash A} g_{u_{2}}^{p} d \mu
\end{aligned}
$$

From this it follows that

$$
\int_{A} g_{u_{2}}^{p} d \mu \leq \int_{A} g_{w}^{p} d \mu \leq \int_{A \cap B} g_{u_{1}}^{p} d \mu+\int_{A \backslash B} g_{v}^{p} d \mu .
$$

This implies that

$$
\begin{aligned}
\int_{\Omega^{\prime}} g_{u}^{p} d \mu & \leq \int_{A} g_{u_{2}}^{p} d \mu+\int_{\Omega^{\prime} \backslash A} g_{u_{1}}^{p} d \mu \\
& \leq \int_{A \cap B} g_{u_{1}}^{p} d \mu+\int_{A \backslash B} g_{v}^{p} d \mu+\int_{\Omega^{\prime} \backslash A} g_{u_{1}}^{p} d \mu \\
& =\int_{B} g_{u_{1}}^{p} d \mu+\int_{A \backslash B} g_{v}^{p} d \mu .
\end{aligned}
$$

Since $u_{1}-\max \left(u_{1}, v\right) \in N^{1, p}\left(\Omega^{\prime}\right), \max \left(u_{1}, v\right) \geq u_{1} \mu$-almost everywhere in $\Omega^{\prime}$, $\max \left(u_{1}, v\right)=u_{1} \mu$-almost everywhere in $\Omega^{\prime} \backslash B$ and $u_{1}$ is a superminimizer, 
we obtain

$$
\begin{aligned}
\int_{B} g_{u_{1}}^{p} d \mu+\int_{\Omega^{\prime} \backslash B} g_{u_{1}}^{p} d \mu & =\int_{\Omega^{\prime}} g_{u_{1}}^{p} d \mu \\
& \leq \int_{\Omega^{\prime}} g_{\max \left(u_{1}, v\right)}^{p} d \mu \leq \int_{B} g_{v}^{p} d \mu+\int_{\Omega^{\prime} \backslash B} g_{u_{1}}^{p} d \mu
\end{aligned}
$$

This implies that

From this we conclude that

$$
\int_{B} g_{u_{1}}^{p} d \mu \leq \int_{B} g_{v}^{p} d \mu
$$

$$
\int_{\Omega^{\prime}} g_{u}^{p} d \mu \leq \int_{B} g_{u_{1}}^{p} d \mu+\int_{A \backslash B} g_{v}^{p} d \mu=\int_{\Omega^{\prime}} g_{v}^{p} d \mu,
$$

and hence $u$ is a superminimizer in $\Omega$.

Next we present a preliminary version of the comparison principle.

3.4. LEMMA. Suppose that $u$ is the solution of the $\mathcal{K}_{\psi, \vartheta}(\Omega)$-obstacle problem and that $\Omega^{\prime} \subset \Omega$ is open. If $v \in N^{1, p}\left(\Omega^{\prime}\right)$ is a minimizer with the boundary values $v$ in $\Omega^{\prime}$ such that $\min (u-v, 0) \in N_{0}^{1, p}\left(\Omega^{\prime}\right)$, then $u \geq v \mu$-almost everywhere in $\Omega^{\prime}$.

Proof. We observe that $\max (u, v)-u=\max (v-u, 0)=-\min (u-v, 0) \in$ $N_{0}^{1, p}\left(\Omega^{\prime}\right)$ and that clearly $\max (u, v) \geq u \mu$-almost everywhere in $\Omega^{\prime}$. By the superminimizing property of $u$, we have

$$
\int_{\Omega^{\prime}} g_{u}^{p} d \mu \leq \int_{\Omega^{\prime}} g_{\max (u, v)}^{p} d \mu \leq \int_{A} g_{v}^{p} d \mu+\int_{\Omega^{\prime} \backslash A} g_{u}^{p} d \mu,
$$

where $A=\left\{x \in \Omega^{\prime}: u(x)<v(x)\right\}$. This implies that

$$
\int_{A} g_{u}^{p} d \mu \leq \int_{A} g_{v}^{p} d \mu
$$

From this we conclude that

$$
\int_{\Omega^{\prime}} g_{\min (u, v)}^{p} d \mu \leq \int_{A} g_{u}^{p} d \mu+\int_{\Omega^{\prime} \backslash A} g_{v}^{p} d \mu \leq \int_{\Omega^{\prime}} g_{v}^{p} d \mu .
$$

Finally we observe that $\min (u, v)-v=\min (u-v, 0) \in N_{0}^{1, p}\left(\Omega^{\prime}\right)$ and that $v$ is the unique minimizer with boundary values $v$ in $\Omega^{\prime}$. It follows that $\min (u, v)=$ $v \mu$-almost everywhere in $\Omega^{\prime}$, and hence $u \geq v \mu$-almost everywhere in $\Omega^{\prime}$.

The following lemma shows that the solution of the obstacle problem is essentially the smallest superminimizer which lies above the obstacle.

3.5. LEMMA. Suppose that $u$ is a solution to the $\mathcal{K}_{\psi, \vartheta}(\Omega)$-obstacle problem. If $v$ is a superminimizer in $\Omega$ such that $v \in \mathcal{K}_{\psi, u}\left(\Omega^{\prime}\right)$ for some open $\Omega^{\prime} \subset \subset \Omega$, then $v \geq u \mu$-almost everywhere in $\Omega^{\prime}$. 
Proof. By Lemma 3.3 the function $\min (u, v)$ is a superminimizer in $\Omega$. Clearly $\min (u, v)-u \in N_{0}^{1, p}\left(\Omega^{\prime}\right)$ and $u \geq \min (u, v) \mu$-almost everywhere in $\Omega^{\prime}$. By the superminimizing property of $\min (u, v)$ in $\Omega^{\prime}$, we have

$$
\int_{\Omega^{\prime}} g_{\min (u, v)}^{p} d \mu \leq \int_{\Omega^{\prime}} g_{u}^{p} d \mu
$$

Since the solution of the obstacle problem is unique, we have $\min (u, v)=u$ $\mu$-almost everywhere in $\Omega^{\prime}$ and consequently $v \geq u \mu$-almost everywhere in $\Omega^{\prime}$.

\section{Regularity of superminimizers}

We are interested in the regularity of superminimizers. Minimizers satisfy Harnack's inequality and are locally Hölder continuous after redefinition on a set of measure zero; see $[\mathrm{KS}]$. In contrast to minimizers, superminimizers are in general not continuous. However, it is possible to obtain some regularity results. Our method is essentially the same as that of $[\mathrm{KS}]$. We begin by recalling the definition of the De Giorgi class.

Let $k_{0} \in \mathbf{R}$ and $0<\rho<R$. A function $u \in N_{\text {loc }}^{1, p}(\Omega)$ belongs to the De Giorgi class $D G_{p}\left(\Omega, k_{0}\right)$, if there is a constant $c<\infty$ such that for all $k \geq k_{0}$ and all $z \in \Omega$ such that $B(z, R) \subset \subset \Omega, u$ satisfies the Caccioppoli type estimate

$$
\int_{A_{z}(k, \rho)} g_{u}^{p} d \mu \leq c(R-\rho)^{-p} \int_{A_{z}(k, R)}(u-k)^{p} d \mu,
$$

where $A_{z}(k, r)=\{x \in B(z, r): u(x)>k\}$ and $g_{u}$ is the minimal $p$-weak upper gradient of $u$ in $\Omega$. If this inequality holds for all $k \in \mathbf{R}$, then we simply write $u \in D G_{p}(\Omega)$.

4.1. LemmA. Suppose that $u$ is a superminimizer in $\Omega$. Then $-u$ belongs to $D G_{p}(\Omega)$.

Proof. Let $B(z, R) \subset \subset \Omega$ and $0<\rho<R$. We show that there is a constant $c<\infty$ such that

$$
\int_{A \rho} g_{u}^{p} d \mu \leq c(R-\rho)^{-p} \int_{A_{R}}(k-u)^{p} d \mu,
$$

where $A_{r}=\{x \in B(z, r): u(x)<k\}$. Let $\eta$ be a $2 /(R-\rho)$-Lipschitz cutoff function such that $0 \leq \eta \leq 1, \eta=1$ on $B(z, \rho)$ and $\eta=0$ on $X \backslash B(z, R)$. We define a test function $v$ by

$$
v=u+\eta \max (k-u, 0) .
$$

Then $u-v \in N_{0}^{1, p}(B(z, R)), v \geq u \mu$-almost everywhere in $B(z, R)$ and $v=u$ $\mu$-almost everywhere in $B(z, R) \backslash A_{R}$. This implies that $v \in \mathcal{K}_{u, u}(B(z, R))$ 
and the superminimizing property of $u$ gives

$$
\int_{A_{\rho}} g_{u}^{p} d \mu \leq \int_{A_{R}} g_{u}^{p} d \mu \leq \int_{A_{R}} g_{v}^{p} d \mu .
$$

On the other hand, we observe that $v=(1-\eta)(u-k)+k$ on $A_{R}$, and hence $g_{v} \leq(k-u) g_{\eta}+(1-\eta) g_{u} \mu$-almost everywhere on $A_{R}$. Since $g_{\eta} \leq 2 /(R-\rho)$ and $\eta=1$ on $B(z, \rho)$, we obtain

$$
\begin{aligned}
\int_{A_{\rho}} g_{u}^{p} d \mu & \leq c \int_{A_{R}}\left((k-u)^{p} g_{\eta}^{p}+(1-\eta)^{p} g_{u}^{p}\right) d \mu \\
& \leq c(R-\rho)^{-p} \int_{A_{R}}(k-u)^{p} d \mu+c \int_{A_{R} \backslash A_{\rho}} g_{u}^{p} d \mu .
\end{aligned}
$$

Adding the term $c \int_{A_{\rho}} g_{u}^{p} d \mu$ to both sides we arrive at the inequality

$$
\int_{A_{\rho}} g_{u}^{p} d \mu \leq \theta \int_{A_{R}} g_{u}^{p} d \mu+c(R-\rho)^{-p} \int_{A_{R}}(k-u)^{p} d \mu
$$

where $\theta=c /(c+1)<1$. For $0<\rho<r \leq R$ the above inequality can be rewritten as

$$
\int_{A_{\rho}} g_{u}^{p} d \mu \leq \theta \int_{A_{r}} g_{u}^{p} d \mu+c(r-\rho)^{-p} \int_{A_{R}}(k-u)^{p} d \mu .
$$

Using a simple iteration argument (see Lemma 5.1 in [Gia]), we obtain (4.2) from the previous inequality.

4.3. LemmA. Let $\psi: \Omega \rightarrow[-\infty, \infty]$ be such that $k_{0}=\operatorname{ess}_{\sup _{\Omega}} \psi<\infty$. Suppose that $u$ is the solution of the $\mathcal{K}_{\psi, \vartheta}(\Omega)$-obstacle problem. Then u belongs to the class $D G_{p}\left(\Omega, k_{0}\right)$.

Proof. Let $B(z, R) \subset \subset \Omega$ and $0<\rho<R$. Set

$$
v=u-\eta \max (u-k, 0)
$$

where $k \geq k_{0}$ and $\eta$ is a cutoff function as in the proof of Lemma 4.1.

Let $x \in \Omega$. If $u(x) \leq k$, then $v(x)=u(x) \geq \psi(x)$. On the other hand, if $u(x)>k$, then

$$
\begin{aligned}
v(x) & =u(x)-\eta(x)(u(x)-k) \\
& =(1-\eta(x))(u(x)-k)+k \\
& \geq k_{0}=\underset{\Omega}{\operatorname{ess} \sup } \psi \geq \psi(x) .
\end{aligned}
$$

From this we conclude that $v \geq \psi \mu$-almost everywhere in $\Omega$ and that $v$ belongs to the class $\mathcal{K}_{\psi, \vartheta}(\Omega)$. Set $A_{r}=A_{z}(k, r)$. Since $v-u \in N_{0}^{1, p}(B(z, R))$ 
and since $g_{v} \leq(u-k) g_{\eta}+(1-\eta) g_{u} \mu$-almost everywhere in $A_{R}$, a similar reasoning as in the proof of Lemma 4.1 gives

$$
\int_{A_{\rho}} g_{u}^{p} d \mu \leq c(R-\rho)^{-p} \int_{A_{R}}(u-k)^{p} d \mu
$$

for every $k \geq k_{0}$, as required.

The solution of an obstacle problem need not be locally bounded above. However, if the obstacle is bounded above, then the solution is locally bounded above. We set $u_{+}=\max (u, 0)$.

4.4. THEOREM. Suppose that $u$ is the solution of the $\mathcal{K}_{\psi, \vartheta}(\Omega)$-obstacle

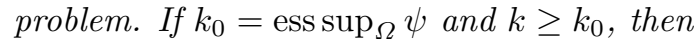

$$
\operatorname{ess~sup}_{B(z, R / 2)} u \leq k+c\left(f_{B(z, R)}(u-k)_{+}^{p} d \mu\right)^{1 / p}
$$

whenever $B(z, R) \subset \subset \Omega$. The constant $c$ depends only on $p$, the doubling constant and the constants in (2.11).

The proof is based on Lemma 4.3 and the De Giorgi method. It is a straightforward adaptation of the argument which proves Theorem 4.2 in [KS], and we leave the details to the interested reader.

4.5. REMARK. It is useful to observe that the claim of Theorem 4.4 holds for every exponent $q>0$. To be more precise, for every $q>0$ there is constant $c$ such that

$$
\operatorname{essip}_{B(z, R / 2)} u \leq k+c\left(f_{B(z, R)}(u-k)_{+}^{q} d \mu\right)^{1 / q} .
$$

This follows from Remark 4.4 (2) in [KS].

Next we derive a local lower bound for superminimizers. We set $u_{-}=$ $-\min (u, 0)$

4.6. THEOREM. Suppose that $u$ is a superminimizer in $\Omega$. Then

$$
\underset{B(z, R)}{\operatorname{essinf}} u \geq-c\left(f_{B(z, 2 R)} u_{-}^{p} d \mu\right)^{1 / p}
$$

whenever $B(z, 2 R) \subset \subset \Omega$. In particular, the function $u$ is bounded from below on every set $\Omega^{\prime} \subset \subset \Omega$. 
Proof. Lemma 4.1 implies that $-u \in D G_{p}(\Omega)$, and hence we can apply Theorem 4.2 in $[\mathrm{KS}]$ to conclude that for every $k \in \mathbf{R}$ we have

$$
-\underset{B(z, R)}{\operatorname{essinf}} u=\underset{B(z, R)}{\operatorname{ess} \sup }(-u) \leq-k+c\left(f_{B(z, 2 R)}(k-u)_{+}^{p} d \mu\right)^{1 / p} .
$$

Choosing $k=0$ we arrive at the desired conclusion.

The next result, called the weak Harnack inequality, follows by Lemma 4.1 immediately from Theorem 7.1 in $[\mathrm{KS}]$.

4.7. LemmA. Suppose that $u \geq 0$ is a superminimizer in an open set $\Omega \subset X$. Then for every ball $B(z, R)$ with $B(z, 5 R) \subset \Omega$ we have

$$
\left(f_{B(z, R)} u^{\sigma} d \mu\right)^{1 / \sigma} \leq c \operatorname{essinf}_{B(z, 3 R)} u
$$

where $c<\infty$ and $\sigma>0$ depend on the data in the same way as in Theorem 4.4 .

\section{Pointwise behaviour of superminimizers}

Next we prove that a superminimizer has a lower semicontinuous representative. We set

$$
\text { ess } \lim _{y \rightarrow x} \inf u(y)=\lim _{r \rightarrow 0} \underset{B(x, r)}{\operatorname{essinf}} u \text {. }
$$

5.1. THEOREM. Suppose that $u$ is a superminimizer in $\Omega$. Then the function $u^{*}: \Omega \rightarrow[-\infty, \infty]$ defined by

$$
u^{*}(x)=\operatorname{ess} \lim _{y \rightarrow x} \inf u(y)
$$

is a lower semicontinuous function in $\Omega$ and belongs to the same equivalence class in $N_{\mathrm{loc}}^{1, p}(\Omega)$ as the function $u$.

Proof. First we note that the function $u^{*}$ defined by (5.2) is automatically lower semicontinuous as an increasing limit of lower semicontinuous functions. Hence it suffices to show that $u$ and $u^{*}$ belong to the same equivalence class in $N_{\text {loc }}^{1, p}(\Omega)$.

Let $\Omega^{\prime} \subset \subset \Omega$ be an open set. Suppose first that $M=\operatorname{essup}_{\Omega^{\prime}} u+1<$ $\infty$. Fix $x \in \Omega^{\prime}$ and let $B(x, 2 R) \subset \subset \Omega^{\prime}$. Set $m(r)=\operatorname{essinf}_{B(x, r)} u$, where $0<r \leq 2 R$. Theorem 4.6 implies that $m(R)>-\infty$. For every $0<5 r \leq R$ the function $u-m(5 r)$ is a superminimizer in $\Omega$ and $u-m(5 r) \geq 0 \mu$-almost everywhere in $B(x, 5 r)$. Hence from Lemma 4.7 we obtain for $0<r<R / 5$ 
that

$$
\begin{aligned}
m(r)-m(5 r) & \geq m(3 r)-m(5 r) \geq c\left(f_{B(x, r)}(u-m(5 r))^{\sigma} d \mu\right)^{1 / \sigma} \\
& \geq c(M-m(5 r))^{(\sigma-1) / \sigma}\left(f_{B(x, r)}(u-m(5 r)) d \mu\right)^{1 / \sigma},
\end{aligned}
$$

where $0<\sigma<1$. This implies that

$$
0 \leq f_{B(x, r)} u d \mu-m(5 r) \leq c(M-m(5 r))^{1-\sigma}(m(r)-m(5 r))^{\sigma}
$$

Since $m(R)>-\infty$, the right hand side tends to zero as $r \rightarrow 0$, and hence

$$
\operatorname{ess} \liminf _{y \rightarrow x} u(y)=\lim _{r \rightarrow 0} f_{B(x, r)} u d \mu .
$$

On the other hand, it follows from the Lebesgue differentiation theorem for Sobolev functions in $[\mathrm{KL}]$ that

$$
\lim _{r \rightarrow 0} f_{B(x, r)} u d \mu=u(x)
$$

for every $x \in \Omega^{\prime} \backslash A$ where $\mathrm{C}_{p}(A)=0$. This implies that $u=u^{*} p$ quasieverywhere in $\Omega^{\prime}$ and that $u$ and $u^{*}$ represent the same function in $N^{1, p}\left(\Omega^{\prime}\right)$.

The general case, when $u$ is possibly not bounded above, follows by truncation. By Lemma 3.3 the function $u_{k}=\min (u, k)$ is a superminimizer in $\Omega$ for each $k=1,2, \ldots$, and by the previous argument $u_{k}^{*}$ is a lower semicontinuous representative of $u_{k}$ in $N_{\text {loc }}^{1, p}(\Omega)$. Moreover, we have

$$
u^{*}(x)=\lim _{k \rightarrow \infty} u_{k}^{*}(x)
$$

for every $x \in \Omega$. Let $\Omega^{\prime} \subset \subset \Omega$ be an open set. Since $u_{k}=u_{k}^{*}$ in $\Omega^{\prime} \backslash A_{k}$ with $\mathrm{C}_{p}\left(A_{k}\right)=0$, we conclude that $u^{*}=u$ in $\Omega^{\prime} \backslash A$, where $A=\bigcup_{k=1}^{\infty} A_{k}$ and

$$
\mathrm{C}_{p}(A)=\mathrm{C}_{p}\left(\bigcup_{k=1}^{\infty} A_{k}\right) \leq \sum_{k=1}^{\infty} \mathrm{C}_{p}\left(A_{k}\right)=0 .
$$

Thus $u^{*}=u p$-quasieverywhere in $\Omega^{\prime}$, and hence $u$ and $u^{*}$ represent the same function in $N^{1, p}\left(\Omega^{\prime}\right)$ as required.

5.4. REMARK. As a byproduct of the proof of Theorem 5.1, we obtain the fact that if $u^{*}$ is the lower semicontinuous representative defined by (5.2) of a superminimizer which is locally bounded above, then

$$
u^{*}(x)=\lim _{r \rightarrow 0} f_{B(x, r)} u^{*} d \mu
$$

for every $x \in \Omega$. 
Next we prove that the solution of the obstacle problem is continuous provided the obstacle is continuous.

5.5. TheOREM. Suppose that $\psi: \Omega \rightarrow[-\infty, \infty)$ is continuous. Then the solution $u$ of the $\mathcal{K}_{\psi, \vartheta}(\Omega)$-obstacle problem is continuous. Moreover, the function $u$ is a minimizer in the open set $\{x \in \Omega: u(x)>\psi(x)\}$.

Proof. We recall that the solution of the obstacle problem is a superminimizer. Let $u$ be the lower semicontinuous representative of the solution given by Theorem 5.1. By (5.2) we observe that it is enough to show that

$$
\text { ess } \limsup _{y \rightarrow x} u(y) \leq u(x)
$$

for every $x \in \Omega$. To this end, let $x \in \Omega$ and $\varepsilon>0$. Then by (5.2) and the fact that $u \in \mathcal{K}_{\psi, \vartheta}(\Omega)$ we have

$$
u(x)=\text { ess } \liminf _{y \rightarrow x} u(y) \geq \text { ess } \liminf _{y \rightarrow x} \psi(y)=\psi(x)
$$

for every $x \in \Omega$, since $\psi$ is continuous.

We observe that $\operatorname{ess} \sup _{B(x, R)} \psi<\infty$ for every $B(x, R) \subset \subset \Omega$, and from Theorem 4.4 we conclude that $\operatorname{ess} \sup _{B(x, R)} u<\infty$ for every $B(x, R) \subset \subset \Omega$. Choose $B(x, R) \subset \subset \Omega$ such that

$$
\sup _{B(x, R)} \psi \leq u(x)+\varepsilon \text { and } \inf _{B(x, R)} u>u(x)-\varepsilon .
$$

This is possible since $u$ is lower semicontinuous and $\psi$ is continuous. Theorem 4.4 and Remark 4.5 imply that

$$
\begin{aligned}
\operatorname{essips}_{B(x, r / 2)}(u-(u(x)+\varepsilon)) & \leq c f_{B(x, r)}(u-(u(x)+\varepsilon))+d \mu \\
& =c f_{B(x, r)}(u-\min (u,(u(x)+\varepsilon))) d \mu
\end{aligned}
$$

for every $0<r<R$. Obviously

$$
\min (u(y), u(x)+\varepsilon) \geq \min \left(\inf _{B(x, r)} u, u(x)+\varepsilon\right) \geq u(x)-\varepsilon
$$

for every $y \in B(x, r)$. Hence

$$
\begin{aligned}
& f_{B(x, r)}(u-\min (u,(u(x)+\varepsilon))) d \mu \\
& \leq f_{B(x, r)}(u-(u(x)-\varepsilon)) d \mu=f_{B(x, r)} u d \mu-u(x)+\varepsilon .
\end{aligned}
$$

Since $u$ is locally bounded above, Remark 5.4 implies that

$$
\lim _{r \rightarrow 0} \int_{B(x, r)} u d \mu=u(x)
$$


and the estimates above imply that

$$
\text { ess } \limsup _{y \rightarrow x} u(y) \leq u(x)+c \varepsilon .
$$

Inequality (5.6) follows by letting $\varepsilon \rightarrow 0$.

Next we prove that $u$ is a minimizer in the open set $A=\{x \in \Omega: u(x)>$ $\psi(x)\}$. Let $\Omega^{\prime} \subset \subset A$ be open and let $v \in N^{1, p}\left(\Omega^{\prime}\right)$ be such that $u-v \in$ $N_{0}^{1, p}\left(\Omega^{\prime}\right)$. We show that

$$
\int_{\Omega^{\prime}} g_{u}^{p} d \mu \leq \int_{\Omega^{\prime}} g_{v}^{p} d \mu
$$

We define $v(x)=u(x)$ when $x \in A \backslash \Omega^{\prime}$.

By [Sh2] there is a continuous function $\widetilde{v}$ in $\Omega^{\prime}$ such that $u-\widetilde{v} \in N_{0}^{1, p}\left(\Omega^{\prime}\right)$ and

$$
\int_{\Omega^{\prime}} g_{\widetilde{v}}^{p} d \mu \leq \int_{\Omega^{\prime}} g_{v}^{p} d \mu+\varepsilon
$$

In fact, the function $u-v$ can be approximated by a Lipschitz continuous function with a compact support in $\Omega^{\prime}$.

Since $u, \widetilde{v}$ and $\psi$ are continuous functions on the compact set $\overline{\Omega^{\prime}}$ and since $u(x)>\psi(x)$ for every $x \in \overline{\Omega^{\prime}}$, there is $t, 0<t<1$, such that

$$
w(x)=u(x)+t(\widetilde{v}(x)-u(x)) \geq \psi(x)
$$

for every $x \in \overline{\Omega^{\prime}}$. Since $w-u=t(\widetilde{v}-u) \in N_{0}^{1, p}\left(\Omega^{\prime}\right)$ and $w(x) \geq \psi(x)$ for every $x \in \Omega^{\prime}$, we conclude that $w \in \mathcal{K}_{u, \psi}\left(\Omega^{\prime}\right)$, and hence by convexity we have

$$
\begin{aligned}
\int_{\Omega^{\prime}} g_{u}^{p} d \mu & \leq \int_{\Omega^{\prime}} g_{w}^{p} d \mu=\int_{\Omega^{\prime}} g_{u+t(\widetilde{v}-u)}^{p} d \mu \\
& \leq \int_{\Omega^{\prime}}\left((1-t) g_{u}+t g_{\widetilde{v}}\right)^{p} d \mu \leq(1-t) \int_{\Omega^{\prime}} g_{u}^{p} d \mu+t \int_{\Omega^{\prime}} g_{\widetilde{v}}^{p} d \mu .
\end{aligned}
$$

This implies that

$$
t \int_{\Omega^{\prime}} g_{u}^{p} d \mu \leq t \int_{\Omega^{\prime}} g_{\widetilde{v}}^{p} d \mu
$$

and since $t>0$ we obtain

$$
\int_{\Omega^{\prime}} g_{u}^{p} d \mu \leq \int_{\Omega^{\prime}} g_{\widetilde{v}}^{p} d \mu \leq \int_{\Omega^{\prime}} g_{v}^{p} d \mu+\varepsilon
$$

The claim follows, since $\varepsilon>0$ was arbitrary.

\section{A convergence result for superminimizers}

We are interested in limits of sequences of superminimizers. We show that the superminimizing property is preserved under increasing or local uniform convergence. 
6.1. Theorem. Suppose that $u_{i}, i=1,2, \ldots$, is an increasing sequence of superminimizers in $\Omega$ and that $u=\lim _{i \rightarrow \infty} u_{i}$ is locally bounded from above in $\Omega$. Then $u$ is a superminimizer in $\Omega$.

Proof. Lemma 4.1 shows that $-u_{i} \in D G_{p}(\Omega), i=1,2, \ldots$ This implies that for every $B(z, R) \subset \subset \Omega$ and $0<\rho<R$ we have

$$
\int_{A_{z}(k, \rho)} g_{u_{i}}^{p} d \mu \leq c(R-\rho)^{-p} \int_{A_{z}(k, R)}\left(-u_{i}-k\right)^{p} d \mu
$$

where $A_{z}(k, r)=\left\{x \in B(z, r):-u_{i}(x)>k\right\}$ and $k \in \mathbf{R}$. For a fixed ball $B(z, R) \subset \subset \Omega$, we choose $k<-\sup _{i} \operatorname{ess}^{\sup _{B(z, R)}} u_{i}$. Then

$$
\int_{B(z, \rho)} g_{u_{i}}^{p} d \mu \leq c(R-\rho)^{-p} \int_{B(z, R)}\left(-u_{1}-k\right)^{p} d \mu
$$

from which we conclude that the sequence $g_{u_{i}}$ is uniformly bounded in $L^{p}\left(\Omega^{\prime}\right)$. This implies that $u \in N_{\mathrm{loc}}^{1, p}(\Omega)$. Moreover, we may assume that the functions $g_{u_{i}}, i=1,2, \ldots$, converge weakly to a function $g_{u}$ in $L^{p}\left(\Omega^{\prime}\right)$, where $g_{u}$ is an upper gradient of $u$.

It remains to show the superminimizing property of $u$. To this end fix an open set $\Omega^{\prime} \subset \subset \Omega$ and a function $v \in N^{1, p}\left(\Omega^{\prime}\right)$ such that $v \geq u \mu$-almost everywhere in $\Omega^{\prime}$ and $v-u \in N_{0}^{1, p}\left(\Omega^{\prime}\right)$. We define $v=u$ in $X \backslash \Omega^{\prime}$. Let $\varepsilon>0$ and choose an open set $\Omega^{\prime \prime}$ such that $\overline{\Omega^{\prime}} \subset \subset \Omega^{\prime \prime} \subset \subset \Omega$ and

$$
\int_{\Omega^{\prime \prime} \backslash \overline{\Omega^{\prime}}} g_{v}^{p} d \mu<\varepsilon
$$

Let $\eta$ be a Lipschitz cutoff function such that $0 \leq \eta \leq 1, \eta=0$ in $\Omega \backslash \Omega^{\prime \prime}$ and $\eta=1$ in $\overline{\Omega^{\prime}}$. For each $i=1,2, \ldots$ set

$$
w_{i}=u_{i}+\eta\left(v-u_{i}\right) .
$$

Then $w_{i} \geq u_{i} \mu$-almost everywhere in $\Omega^{\prime \prime}, w_{i}-u_{i} \in N_{0}^{1, p}\left(\Omega^{\prime \prime}\right)$, and by Lemma 2.4 we have

$$
g_{w_{i}} \leq(1-\eta) g_{u_{i}}+\eta g_{v}+\left(v-u_{i}\right) g_{\eta}
$$

$\mu$-almost everywhere in $\Omega^{\prime \prime}$.

The superminimizing property of $u_{i}$ implies that

$$
\begin{aligned}
& \left(\int_{\Omega^{\prime \prime}} g_{u_{i}}^{p} d \mu\right)^{1 / p} \leq\left(\int_{\Omega^{\prime \prime}} g_{w_{i}}^{p} d \mu\right)^{1 / p} \\
& \quad \leq\left(\int_{\Omega^{\prime \prime}}\left((1-\eta) g_{u_{i}}+\eta g_{v}\right)^{p} d \mu\right)^{1 / p}+\left(\int_{\Omega^{\prime \prime}}\left(v-u_{i}\right)^{p} g_{\eta}^{p} d \mu\right)^{1 / p} \\
& \quad=\alpha_{i}+\beta_{i}
\end{aligned}
$$


We now use the following elementary inequality: If $\alpha, \beta \geq 0$, then

$$
(\alpha+\beta)^{p} \leq \alpha^{p}+p \beta(\alpha+\beta)^{p-1} .
$$

Together with (6.4) this yields

$$
\begin{aligned}
\int_{\Omega^{\prime \prime}} g_{u_{i}}^{p} d \mu & \leq \int_{\Omega^{\prime \prime}}\left((1-\eta) g_{u_{i}}+\eta g_{v}\right)^{p} d \mu+p \beta_{i}\left(\alpha_{i}+\beta_{i}\right)^{p-1} \\
& \leq \int_{\Omega^{\prime \prime}}(1-\eta) g_{u_{i}}^{p} d \mu+\int_{\Omega^{\prime \prime}} \eta g_{v}^{p} d \mu+p \beta_{i}\left(\alpha_{i}+\beta_{i}\right)^{p-1}
\end{aligned}
$$

where we also used the convexity of the function $t \mapsto t^{p}$. This implies that

$$
\begin{aligned}
\int_{\overline{\Omega^{\prime}}} g_{u_{i}}^{p} d \mu & \leq \int_{\Omega^{\prime \prime}} \eta g_{u_{i}}^{p} d \mu \leq \int_{\Omega^{\prime \prime}} \eta g_{v}^{p} d \mu+p \beta_{i}\left(\alpha_{i}+\beta_{i}\right)^{p-1} \\
& \leq \int_{\overline{\Omega^{\prime}}} g_{v}^{p} d \mu+\varepsilon+p \beta_{i}\left(\alpha_{i}+\beta_{i}\right)^{p-1}
\end{aligned}
$$

where we used (6.2) in the last inequality.

Since $g_{u_{i}} \rightarrow g_{u}$ weakly in $L^{p}\left(\overline{\Omega^{\prime}}\right)$, the lower semicontinuity of the $L^{p}$-norm implies that

$$
\int_{\overline{\Omega^{\prime}}} g_{u}^{p} d \mu \leq \liminf _{i \rightarrow \infty} \int_{\overline{\Omega^{\prime}}} g_{u_{i}}^{p} d \mu .
$$

Recall that $g_{\eta_{i}}=0 \mu$-almost everywhere in $\overline{\Omega^{\prime}}$ and $v=u$ in $\Omega \backslash \Omega^{\prime}$. Since $u_{i} \rightarrow u$ in $L_{\text {loc }}^{p}(\Omega)$, we conclude that

$$
\beta_{i}=\left(\int_{\Omega^{\prime \prime} \backslash \Omega^{\prime}}\left(u-u_{i}\right)^{p} g_{\eta}^{p} d \mu\right)^{1 / p} \rightarrow 0
$$

as $i \rightarrow \infty$. Since the sequence $\alpha_{i}, i=1,2, \ldots$, is bounded, letting $i \rightarrow \infty$ in (6.5) and using (6.6), we obtain

$$
\int_{\overline{\Omega^{\prime}}} g_{u}^{p} d \mu \leq \int_{\overline{\Omega^{\prime}}} g_{v}^{p} d \mu+\varepsilon .
$$

Now $u=v \mu$-almost everywhere on $\partial \Omega^{\prime}$ and hence $g_{u}=g_{v} \mu$-almost everywhere on $\partial \Omega^{\prime}$. Hence the above inequality holds on $\Omega^{\prime}$ as well, and since $\varepsilon>0$ was arbitrary, this proves the required minimizing property of $u$.

6.7. Remarks. (1) Suppose that $u_{i}, i=1,2, \ldots$, are as in the statement of Theorem 6.1 and that instead of requiring that the sequence is bounded from above we assume that the limit function $u$ is in $N_{\text {loc }}^{1, p}(\Omega)$. Then $u$ is a superminimizer. This follows easily from Theorem 6.1 and from the fact that $u \in N_{\text {loc }}^{1, p}(\Omega)$ is a superminimizer if and only if $\min (u, k)$ is a superminimizer for every $k \in \mathbf{R}$.

(2) It is easy to modify the previous proof to yield the following result: If the sequence $u_{i}$ of minimizers or superminimizers converges locally uniformly 
in $\Omega$, then the limit function $u=\lim _{i \rightarrow \infty} u_{i}$ is a minimizer or a superminimizer in $\Omega$, respectively.

\section{Superharmonic functions}

In this section we define superharmonic functions and study their basic properties.

Let $\Omega$ be an open subset of $X$. A function $v: \Omega \rightarrow \mathbf{R}$ is p-harmonic, or simply harmonic, in $\Omega$ if it is a continuous minimizer in $\Omega$. By [KS], every minimizer is locally Hölder continuous after redefinition on a set of measure zero.

Suppose that $\Omega^{\prime} \subset \subset \Omega$ is open and $v \in N^{1, p}\left(\Omega^{\prime}\right)$. Let $h_{\Omega^{\prime}}(v)=h(v)$ be the unique harmonic function in $\Omega^{\prime}$ with $v-h(v) \in N_{0}^{1, p}\left(\Omega^{\prime}\right)$. Observe that $h(v)$ exists by Theorem 3.2 and by the results of $[\mathrm{KS}]$.

A function $u: \Omega \rightarrow(-\infty, \infty]$ is called p-superharmonic, or simply superharmonic, in $\Omega$, if it satisfies the following properties:

(i) $u$ is lower semicontinuous in $\Omega$;

(ii) $u$ is not identically $\infty$ in any component of $\Omega$;

(iii) for every open $\Omega^{\prime} \subset \subset \Omega$ the following comparison principle holds: if $v \in C\left(\overline{\Omega^{\prime}}\right) \cap N^{1, p}\left(\Omega^{\prime}\right)$ and $v \leq u$ on $\partial \Omega^{\prime}$, then $h(v) \leq u$ in $\Omega^{\prime}$.

A function $u$ is subharmonic in $\Omega$, if $-u$ is superharmonic in $\Omega$.

We begin with some elementary observations. If $u$ is superharmonic, then $\alpha u$ and $u+\beta$ are superharmonic when $\alpha \geq 0$ and $\beta \in \mathbf{R}$. Moreover, the minimum of two superharmonic functions is superharmonic. However, the sum of two superharmonic function is in general not superharmonic.

We next observe that the class of superharmonic functions is closed under increasing or local uniform convergence.

7.1. Lemma. Suppose that $u_{i}, i=1,2, \ldots$, is a sequence of superharmonic functions in $\Omega$. If the sequence $u_{i}$ is either increasing or converges locally uniformly, then the limit function $u=\lim _{i \rightarrow \infty} u_{i}$ is superharmonic unless $u$ is identically $\infty$ in any component of $\Omega$.

Proof. We consider only the case when the sequence is increasing; the other case is easier. It suffices to check the comparison principle (iii) in the definition. Fix an open set $\Omega^{\prime} \subset \subset \Omega$ and let $v \in C\left(\overline{\Omega^{\prime}}\right) \cap N^{1, p}\left(\Omega^{\prime}\right)$ with $v \leq u$ on $\partial \Omega^{\prime}$. Let $\varepsilon>0$. Since $u_{i}, i=1,2, \ldots$, are lower semicontinuous and $v$ is continuous, the sets

$$
A_{i}=\left\{x \in \partial \Omega^{\prime}: v(x)<u_{i}(x)+\varepsilon\right\},
$$

$i=1,2, \ldots$, are open in $\partial \Omega^{\prime}$ and $A_{1} \subset A_{2} \subset \cdots$. Since $\partial \Omega^{\prime} \subset \bigcup_{i=1}^{\infty} A_{i}$ and $\partial \Omega^{\prime}$ is compact, there is $i_{0}$ such that $\partial \Omega^{\prime} \subset A_{i}$ for $i \geq i_{0}$. Since $u_{i}+\varepsilon$, $i=1,2, \ldots$, are superharmonic, by the comparison principle we have $h(v) \leq$ 
$u_{i}+\varepsilon, i \geq i_{0}$, in $\Omega^{\prime}$, and consequently $h(v) \leq u+\varepsilon$ in $\Omega^{\prime}$. This implies that $h(v) \leq u$ in $\Omega^{\prime}$ as required.

The following version of the comparison principle is very useful in nonlinear potential theory.

7.2. TheOREM. Suppose that $u$ is superharmonic and that $v$ is subharmonic in $\Omega$. If

$$
\limsup _{y \rightarrow x} v(y) \leq \liminf _{y \rightarrow x} u(y)
$$

for every $x \in \partial \Omega$, and if both sides of (7.3) are not simultaneously $\infty$ or $-\infty$, then $v \leq u$ in $\Omega$.

Proof. Let $\varepsilon>0$. Let $\Omega^{\prime}$ be an open set such that $\Omega^{\prime} \subset \subset \Omega$ and $v<$ $u+\varepsilon$ on $\partial \Omega^{\prime}$. Since $v$ is upper semicontinuous in $\Omega$, there is a decreasing sequence $\varphi_{i}, i=1,2, \ldots$, of Lipschitz continuous functions in $\overline{\Omega^{\prime}}$ such that $v=\lim _{i \rightarrow \infty} \varphi_{i}$ everywhere in $\overline{\Omega^{\prime}}$. Since $\partial \Omega^{\prime}$ is compact and $u+\varepsilon$ is lower semicontinuous, there is $i_{0}$ such that $\varphi_{i} \leq u+\varepsilon$ on $\partial \Omega^{\prime}$ when $i \geq i_{0}$. Since $\varphi_{i} \in C\left(\overline{\Omega^{\prime}}\right) \cap N^{1, p}\left(\Omega^{\prime}\right), i=1,2, \ldots$, and $u+\varepsilon$ is superharmonic, by the comparison principle we conclude that $h\left(\varphi_{i}\right) \leq u+\varepsilon$ in $\Omega^{\prime}$ for $i \geq i_{0}$. On the other hand, $\varphi_{i} \geq v$ on $\partial \Omega^{\prime}$, and since $v$ is subharmonic we have $h\left(\varphi_{i}\right) \geq v$ in $\Omega^{\prime}$. This implies that $v \leq h\left(\varphi_{i}\right) \leq u+\varepsilon$ in $\Omega^{\prime}$ when $i \geq i_{0}$, and by letting $\varepsilon \rightarrow 0$ we obtain $v \leq u$ in $\Omega^{\prime}$. The claim follows from this, since $\Omega$ can be exhausted by sets $\Omega^{\prime} \subset \subset \Omega$ with the required properties.

Next we study the connection between superminimizers and superharmonic functions.

7.4. Proposition. If $u$ is a superminimizer in $\Omega$ such that

$$
u(x)=\operatorname{ess} \lim _{y \rightarrow x} \inf u(y)
$$

for every $x \in \Omega$, then $u$ is superharmonic.

Proof. The function $u$ is lower semicontinuous by (7.5). Since $u$ is lower semicontinuous, it is bounded from below on every set $\Omega^{\prime} \subset \subset \Omega$. Since $u$ is in $N_{\text {loc }}^{1, p}(\Omega)$, it is finite $\mu$-almost everywhere on $\Omega$ and hence cannot be identically $\infty$ in any component of $\Omega$.

Let $\Omega^{\prime}$ be an open set such that $\Omega^{\prime} \subset \subset \Omega$ and suppose that $v \in C\left(\overline{\Omega^{\prime}}\right) \cap$ $N^{1, p}\left(\Omega^{\prime}\right)$ with $v \leq u$ on $\partial \Omega^{\prime}$. Then $\min (u-v, 0) \in N_{0}^{1, p}\left(\Omega^{\prime}\right)$, and hence we have $\min (u-h(v), 0) \in N_{0}^{1, p}\left(\Omega^{\prime}\right)$. Since $h(v)$ is a minimizer in $\Omega^{\prime}$, Lemma 3.4 implies that $u \geq h(v) \mu$-almost everywhere in $\Omega^{\prime}$, and finally (7.5) implies that $u \geq h(v)$ everywhere in $\Omega^{\prime}$. 
The following corollary is an immediate consequence of Proposition 7.4 and the fact that every superminimizer has a lower semicontinuous representative; see Theorem 5.1.

7.6. COROllary. For every superminimizer $u$ in $\Omega$ there is a superharmonic function $v$ such that $u=v \mu$-almost everywhere in $\Omega$.

Suppose that $u_{i}, i=1,2, \ldots$, is an increasing sequence of lower semicontinuous superminimizers in $\Omega$ given by Theorem 5.1. By Proposition 7.4 every $u_{i}$ is superharmonic and Lemma 7.1 implies that the limit function $u=\lim _{i \rightarrow \infty} u_{i}$ is superharmonic unless it is identically infinity in some component of $\Omega$. We now show that, conversely, every superharmonic function can be locally approximated from below by superminimizers. It is important for us that the approximating superminimizers can be chosen to be continuous.

7.7. TheOREM. Suppose that $u$ is a superharmonic function in $\Omega$ and let $\Omega^{\prime}$ be an open set such that $\Omega^{\prime} \subset \subset \Omega$. Then there is an increasing sequence of continuous superminimizers $u_{i}, i=1,2, \ldots$, in $\Omega^{\prime}$ such that $u=\lim _{i \rightarrow \infty} u_{i}$ everywhere in $\Omega^{\prime}$.

Proof. Let $\Omega^{\prime}$ be an open set such that $\Omega^{\prime} \subset \subset \Omega$. Since $u$ is lower semicontinuous in $\Omega$, there is an increasing sequence $\varphi_{i}, i=1,2, \ldots$, of Lipschitz continuous functions in $\overline{\Omega^{\prime}}$ such that $u=\lim _{i \rightarrow \infty} \varphi_{i}$ everywhere in $\overline{\Omega^{\prime}}$. Let $u_{i}$, $i=1,2, \ldots$, be the solution to the $\mathcal{K}_{\varphi_{i}, \varphi_{i}}\left(\Omega^{\prime}\right)$-obstacle problem in $\Omega^{\prime}$. Each $u_{i}$ is a superminimizer and we will show that $u_{i}, i=1,2, \ldots$, is the required sequence. By Theorem 5.5 the functions $u_{i}, i=1,2, \ldots$, are continuous in $\Omega^{\prime}$ and the sets $A_{i}=\left\{x \in A: u_{i}(x)>\varphi_{i}(x)\right\}, i=1,2, \ldots$, are open. Moreover, $u_{i}-\varphi_{i} \in N_{0}^{1, p}\left(A_{i}\right)$ and by Theorem 5.5 the functions $u_{i}, i=1,2, \ldots$, are minimizers in $A_{i}$ with boundary values $\varphi_{i}$, and hence $u_{i}=h\left(\varphi_{i}\right), i=1,2, \ldots$, in $A_{i}$. Since $\varphi_{i}$ is continuous in $\overline{A_{i}}$ and $\varphi_{i} \leq u_{i}$ on $\partial A_{i}$, the comparison principle yields $u_{i}=h\left(\varphi_{i}\right) \leq u$ in $A_{i}$. Since $u_{i} \leq \varphi_{i} \leq u$ in $\Omega^{\prime} \backslash A_{i}$, it follows that $u_{i} \leq u$ in $\Omega^{\prime}$. By continuity, $u_{i} \geq \varphi_{i}$ in $\Omega^{\prime}$ and we obtain

$$
u=\lim _{i \rightarrow \infty} \varphi_{i} \leq \lim _{i \rightarrow \infty} u_{i} \leq u
$$

in $\Omega^{\prime}$.

Since $\min \left(u_{i}, u_{i+1}\right) \in \mathcal{K}_{\varphi_{i}, u_{i}}\left(\Omega^{\prime}\right)$ and $\min \left(u_{i}, u_{i+1}\right)$ is a superminimizer in $\Omega^{\prime}$, by Lemma 3.5 we conclude that $u_{i+1} \geq u_{i} \mu$-almost everywhere in $\Omega^{\prime}$ and by continuity everywhere in $\Omega^{\prime}$. This shows that the sequence $u_{i}, i=1,2, \ldots$, is increasing and the proof is complete.

7.8. Corollary. If $u$ is superharmonic in $\Omega$ and, in addition, $u$ is locally bounded above, then $u \in N_{\mathrm{loc}}^{1, p}(\Omega)$ and $u$ is a superminimizer. 
Proof. By Theorem 6.1 the limit of a locally bounded and increasing sequence of superminimizers is a superminimizer. Thus the claim follows from Theorem 7.7 .

A superminimizer belongs to $N_{\text {loc }}^{1, p}(\Omega)$ by definition. Corollary 7.6 shows that every superminimizer has a superharmonic representative. We now show that, conversely, if $u$ is a superharmonic function which belongs to $N_{\mathrm{loc}}^{1, p}(\Omega)$, then it is a superminimizer.

7.9. Corollary. If $u$ is superharmonic and, in addition, $u \in N_{\operatorname{loc}}^{1, p}(\Omega)$, then $u$ is a superminimizer.

Proof. Since $u$ is superharmonic, by Theorem 7.7 it is locally a limit of an increasing sequence of superminimizers. If the limit function $u$ belongs to $N_{\text {loc }}^{1, p}(\Omega)$, then it is a superminimizer by Remark $6.7(1)$.

Proposition 7.4 implies, in particular, that harmonic functions are both super- and subharmonic. The following lemma provides a converse to this result.

7.10. Corollary. A function is harmonic in $\Omega$ if and only if it is both superharmonic and subharmonic in $\Omega$.

Proof. Assume that $u$ is both super- and subharmonic in $\Omega$. Then $u$ is continuous in $\Omega$ and hence locally bounded in $\Omega$. Corollary 7.8 implies that $u$ is both a superminimizer and a subminimizer in $\Omega$, and consequently it is a minimizer in $\Omega$.

Observe that there is no assumption on the integrability of a superharmonic function. The following lemma shows that a superharmonic function is locally integrable to a small power.

7.11. LemmA. Suppose that $u \geq 0$ is superharmonic in $\Omega$. Then for every ball $B(z, R)$ with $B(z, 5 R) \subset \Omega$ we have

$$
\left(f_{B(z, R)} u^{\sigma} d \mu\right)^{1 / \sigma} \leq c \inf _{B(z, 3 R)} u
$$

where $c<\infty$ and $\sigma>0$ depend on the data in the same way as in Lemma 4.7.

7.13. REMARK. Since lower semicontinuous functions are locally bounded from below, and superharmonic functions are not identically infinity, by adding a constant to the function it follows from (7.12) that every superharmonic function is locally integrable to a small power $\sigma>0$ and, in particular, every superharmonic function is finite $\mu$-almost everywhere. 
Proof of Lemma 7.11. Let $z \in \Omega$ be such that $u(z)<\infty$, and let $\Omega^{\prime} \subset \subset \Omega$ be such that $B(z, 5 R) \subset \subset \Omega^{\prime}$. Let $u_{i}, i=1,2, \ldots$, be an increasing sequence of continuous superminimizers in $\Omega^{\prime}$ such that $u=\lim _{i \rightarrow \infty} u_{i}$ in $\Omega^{\prime}$. Then $\inf _{B(z, R)} u<\infty$ whenever $R>0$ is such that $B(z, R) \subset \Omega^{\prime}$. By Lemma 4.7 we have for every ball $B(z, R)$ with $B(z, 5 R) \subset \Omega^{\prime}$

$$
\left(f_{B(z, R)} u_{i}^{\sigma} d \mu\right)^{1 / \sigma} \leq c \inf _{B(z, 3 R)} u_{i} \leq c \inf _{B(z, 3 R)} u,
$$

where $c<\infty$ and $\sigma>0$ are as in Lemma 4.7. In particular, $c$ and $\sigma$ are independent of $i$. Letting $i \rightarrow \infty$ we conclude that

$$
\left(f_{B(z, R)} u^{\sigma} d \mu\right)^{1 / \sigma} \leq c \inf _{B(z, 3 R)} u .
$$

Next we show that if $u$ is a superminimizer in $\Omega$, then $u$ is a superharmonic in $\Omega$ if and only if $u(x)=\operatorname{ess}_{\liminf } \inf _{y \rightarrow x} u(y)$ for every $x \in \Omega$.

7.14. TheOrem. If $u$ is superharmonic in $\Omega$, then

$$
u(x)=\operatorname{ess} \liminf _{y \rightarrow x} u(y)
$$

for every $x \in \Omega$.

As a corollary we obtain the following uniqueness result.

7.15. Corollary. If $u$ and $v$ are superharmonic functions in $\Omega$ and $u=v \mu$-almost everywhere in $\Omega$, then $u=v$ everywhere in $\Omega$.

The proof of Theorem 7.14 is based on the following lemma.

7.16. Lemma. Suppose that $u$ is a superharmonic function in $\Omega$ such that $u=0 \mu$-almost everywhere in $\Omega$. Then $u=0$ everywhere in $\Omega$.

Proof. Since $u$ is lower semicontinuous, we conclude that

$$
u(x) \leq \liminf _{y \rightarrow x} u(y) \leq 0
$$

for every $x \in \Omega$. On the other hand, $v=\min (u, 0)$ is a superharmonic function which is bounded from above. By Corollary 7.8 it is a superminimizer and by Theorem 4.6 we have

$$
\inf _{B(z, R)} v \geq-c\left(f_{B(z, 2 R)} v_{-}^{p} d \mu\right)^{1 / p}
$$

whenever $B(z, 2 R) \subset \subset \Omega$. Since $u(x)=0$ for $\mu$-almost every $x \in \Omega$, we conclude that $\inf _{B(z, R)} v \geq 0$ and hence $u(x) \geq 0$ for every $x \in B(z, R)$. 
Proof of Theorem 7.14. Let $x \in \Omega$. Since $u$ is lower semicontinuous, we have

$$
u(x) \leq \liminf _{y \rightarrow x} u(y) \leq \operatorname{ess} \liminf _{y \rightarrow x} u(y) .
$$

Hence it is enough to show that

$$
\lambda=\operatorname{ess} \lim _{y \rightarrow x} \inf u(y) \leq u(x) .
$$

For every $\varepsilon>0$ there is $r>0$ such that $u(y)>\lambda-\varepsilon$ for $\mu$-almost every $y \in$ $B(x, r)$, where $B(x, r) \subset \Omega$. Then $v=\min (u, \lambda-\varepsilon)-(\lambda-\varepsilon)$ is superharmonic in $\Omega$ and $v=0 \mu$-almost everywhere in $B(x, r)$. By Lemma 7.16 we conclude that $u(y) \geq \lambda-\varepsilon$ for every $y \in B(x, r)$. In particular, $u(x) \geq \lambda-\varepsilon$. Since $\varepsilon>0$ was arbitrary, we have established that $\lambda \leq u(x)$.

\section{REFERENCES}

[B] J. Björn, Boundary continuity for quasiminimizers on metric spaces, Illinois J. Math. 46 (2002), 383-403.

[BBS] A. Björn, J. Björn, and N. Shanmugalingam, The Dirichlet problem for p-harmonic functions on metric measure spaces, preprint (2001).

[BMS] J. Björn, P. MacManus, and N. Shanmugalingam, Fat sets and pointwise boundary estimates for p-harmonic functions in metric spaces, J. Anal. Math. 85 (2001), 339-369.

[C] J. Cheeger, Differentiability of Lipschitz functions on metric measure spaces, Geom. Funct. Anal. 9 (1999), 428-517.

[Gia] M. Giaquinta, Introduction to regularity theory for nonlinear elliptic systems, Birkhäuser Verlag, Zürich, 1993.

[Giu] E. Giusti, Metodi diretti nel calcolo delle variazioni, Unione Matematica Italiana, Bologna, 1994.

[Ha] P. Hajłasz, Sobolev spaces on an arbitrary metric space, Potential Anal. 5 (1995), 403-415.

[HaK $]$ P. Hajłasz and P. Koskela, Sobolev met Poincaré, Mem. Amer. Math. Soc. 145 (2000).

[He] J. Heinonen, Lectures on analysis on metric spaces, Springer-Verlag, New York, 2001.

[HKM] J. Heinonen, T. Kilpeläinen, and O. Martio, Nonliear potential theory of degenerate elliptic equations, Oxford University Press, Oxford, 1993.

[HeK] J. Heinonen and P. Koskela, Quasiconformal maps in metric spaces with controlled geometry, Acta Math. 181 (1998), 1-61.

$[\mathrm{KKM}]$ T. Kilpeläinen, J. Kinnunen, and O. Martio, Sobolev spaces with zero boundary values on metric spaces, Potential Anal. 12 (2000), 233-247.

[KL] J. Kinnunen and V. Latvala, Lebesgue points for Sobolev functions on metric spaces, to appear in Revista Mat. Iberoamericana.

[KM] J. Kinnunen and O. Martio, Sobolev capacity on metric spaces, Ann. Acad. Sci. Fenn. Math. 21 (1996), 367-382.

[KS] J. Kinnunen and N. Shanmugalingam, Regularity of quasi-minimizers on metric spaces, Manuscripta Math. 105 (2001), 401-423.

[Sh1] N. Shanmugalingam, Newtonian spaces: An extension of Sobolev spaces to metric measure spaces, Revista Mat. Iberoamericana 16 (2000), 243-279.

[Sh2] _ Harmonic functions on metric spaces, Illinois J. Math. 45 (2001), 10211050. 
Juha Kinnunen, Institute of Mathematics, P.O. Box 1100, Fin-02015 Helsinki University of Technology, Finland

E-mail address: juha.kinnunen@hut.fi

Olli Martio, Department of Mathematics, P.O. Box 4, Fin-00014 University of Helsinki, Finland

E-mail address: olli.martio@helsinki.fi 